\title{
Focused Assessment with Sonography for Urinary Schistosomiasis (FASUS)_pilot evaluation of a simple point-of-care ultrasound protocol and short training program for detecting urinary tract morbidity in highly endemic settings
}

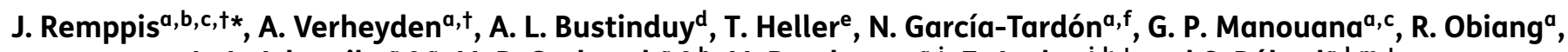
A. A. Adegnika ${ }^{a, c, g}$, M. P. Grobusch ${ }^{a, c, h}$, M. Ramharter ${ }^{a, i}, E^{2}$ Joekes $^{j, k, t}$ and S. Bélard ${ }^{a, l, m, t}$

${ }^{a}$ Centre de Recherches Médicales de Lambaréné (CERMEL), B.P: 242 Lambaréné, Gabon;

${ }^{b}$ Department of Haematology and Oncology, Children's University Hospital, Hoppe-Seyler-Straße 1, 72076 Tübingen, Germany;

'Institute of Tropical Medicine, University of Tübingen, Wilhelmstraße 27, 72074 Tübingen, Germany;

${ }^{d}$ Department of Clinical Research, London School of Hygiene and Tropical Medicine, Keppel Street, London, WC1E 7HT, UK;

e Lighthouse Clinic, Kamuzu Central Hospital, Area 33 Mzimba Street. P.O. Box 106, Lilongwe, Malawi;

fClinical Chemistry Laboratory, Isala, Dr. van Heesweg 2, 8025 AB Zwolle, the Netherlands;

${ }^{9}$ German Center for Infection Research, Wilhelmstraße 27, 72074 Tübingen, Germany;

${ }^{h}$ Center of Tropical Medicine and Travel Medicine, Amsterdam University Medical Centers, location AMC, University of Amsterdam, Meibergdreef 9, 1105 AZ Amsterdam, the Netherlands;

'Department of Tropical Medicine, Bernhard Nocht Institute for Tropical Medicine \& I Department of Medicine University Medical Center Hamburg-Eppendorf, Bernhard-Nocht-Straße 74, 20359 Hamburg, Germany;

'Liverpool School of Tropical Medicine, Pembroke Pl, Liverpool L3 5QA, UK;

k'Department of Radiology, Royal Liverpool University Hospital NHS Trust, Prescot St, Liverpool L7 8XP, UK;

Department of Paediatric Pulmonology, Immunology and Intensive Care Medicine, Charité - Universitätsmedizin Berlin, Augustenburgerplatz 1, 10117 Berlin, Germany;

mBerlin Institute of Health, Anna-Louisa-Karsch-Straße 2, 10178 Berlin, Germany

*Corresponding author: Tel: +49 1638698 219; Email: jonathan.remppis@posteo.de

tThe authors wish it to be known that, in their opinion, the first two authors should be regarded as joint First Authors and the last two authors as joint Last Authors.

Received 18 March 2019; revised 19 August 2019; editorial decision 25 August 2019; accepted 5 September 2019

Background: Urogenital schistosomiasis (UGS) causes inflammation and fibrosis of the urinary tract. In resourcelimited settings, affordable tools for morbidity assessment in clinical care are needed. Point-of-care ultrasound has not yet been validated for UGS-related pathology.

Methods: We developed a protocol for Focused Assessment with Sonography for Urinary Schistosomiasis (FASUS), assessing pathology of the bladder wall, ureters and kidneys. Following standardized training, two clinicians performed FASUS on children and adults with hematuria in Lambaréné, Gabon. Recorded ultrasound clips were remotely reviewed by two ultrasound experts as a diagnostic reference.

Results: In 2015 and 2016, scans were performed in 118 patients. The image quality was sufficient in 90\% of bladder views and more than $97 \%$ of kidney views. UGS-compatible pathology was detected in 51/118 (43\%) by the operator and in 46/107 (43\%) by the experts among baseline scans of sufficient quality. Inter-rater agreement between operators and experts was very good $(\kappa>0.8)$ for hydronephrosis and good $(\kappa>0.6)$ for bladder wall thickening.

Conclusions: FASUS is a promising clinical, point-of-care tool for detecting UGS-related urinary tract morbidity in symptomatic patients. Based on larger validation studies, appropriate diagnostic and therapeutic algorithms for the use of FASUS should be established.

(C) The Author(s) 2019. Published by Oxford University Press on behalf of Royal Society of Tropical Medicine and Hygiene. All rights reserved. For permissions, please e-mail: journals.permissions@oup.com.

This article is published and distributed under the terms of the Oxford University Press, Standard Journals Publication Model (https://academi c.oup.com/journals/pages/open_access/funder_policies/chorus/standard_publication_model) 
Keywords: diagnostic imaging, Gabon, hydronephrosis, point-of-care testing, Schistosoma haematobium, tropical medicine

\section{Introduction}

Urogenital schistosomiasis (UGS), a waterborne disabling parasitic neglected tropical disease, affects an estimated 112 million people, with more than 400 million people at risk. ${ }^{1,2}$ Chronic infection and its sequelae cause substantial morbidity reported mainly, but not exclusively, in school-aged children and young adults. ${ }^{3,4}$ UGS-related pathology may include bladder wall thickening and/or distal ureteric wall thickening. At an advanced stage it may include upper urinary tract obstruction and renal failure, ${ }^{5}$ as well as squamous cell carcinoma of the bladder. ${ }^{6}$ If treated early in the course of disease, a certain proportion of the pathological changes in the urinary tract are reversible. ${ }^{3}$ In patients with irreversible damage, options for treatment will depend on the type of damage and available treatment.

In most high- and moderate-prevalence areas the only available diagnostic tools are detection of hematuria by urine dipstick and direct parasitological examination of the urine. These tools, as well as novel methods such as detection of specific antibodies, antigens or genetic material, ${ }^{7-9}$ can confirm infection but detect neither anatomical changes due to the infection nor sequelae after treatment.

In prevalence surveys, ultrasound has long been established as an effective imaging tool to assess urinary tract morbidity in UGS and to monitor treatment response, ${ }^{10}$ with results which are comparable with more invasive methods such as cystoscopy. ${ }^{11}$ As ultrasound is a test for morbidity from UGS, and not for active infection, it complements, rather than replaces, parasitological tests.

In 1996, the WHO published a standardized protocol for the ultrasonographic assessment of schistosomiasis-related morbidity, commonly known as the Niamey protocol. ${ }^{12}$ However, this protocol is specifically designed for application in large-scale control programs and not for diagnosis in the clinical setting. It includes a detailed and relatively complex grading system, which is less relevant to clinical application. ${ }^{10}$ Contrary to widespread experience of using ultrasound in prevalence surveys, it has not been validated for routine patient care in endemic areas. In addition, prevalence surveys mostly rely on operators with professional ultrasound training. In many clinical settings, this expertise is lacking.

Point-of-care ultrasound (POCUS) has been successfully implemented for various other indications in tropical medicine, e.g. HIV-associated TB and echinococcosis. ${ }^{13}$

The aim of this study was to pilot a POCUS protocol for detection of UGS-related urinary tract morbidity in symptomatic patients, to evaluate practical feasibility and evaluate the performance of operators with limited training, using low-cost equipment.

\section{Methods}

This study was conducted at the Centre de Recherches Médicales de Lambaréné (CERMEL) located in the semi-rural setting of Lambaréné, Gabon, where UGS is highly prevalent. ${ }^{14-19}$ It was part of a larger study, which additionally assessed epidemiological aspects as well as immunological and metabolomic aspects of UGS; these will be reported separately. Procedures relevant for the pilot assessment of the POCUS protocol were: (1) development of a POCUS scan protocol; (2) the training of two operators; (3) evaluation of the diagnostic accuracy of POCUS operators, using remote expert image review as a reference; and (4) comparing the ultrasound findings with parasitology test results.

\section{Protocol development}

A simple and easy-to-learn protocol was designed by the study team, selecting clinically relevant criteria from the Niamey proto$\mathrm{col}$. The Niamey protocol provides standards for the evaluation of the urinary bladder (shape, bladder wall thickening, irregularities and polyps), ureters (ureteral dilation), renal pelvis (degree of hydronephrosis), along with several additional evaluations (bladder volume, residual urine after voiding and fibrosis of the renal pelvis). Final evaluation includes multiple measurements for the calculation of a final severity score. ${ }^{12}$ Based on the nomenclature of previous POCUS techniques ${ }^{13}$ we named our protocol: 'Focused Assessment with Sonography for Urinary Schistosomiasis' (FASUS). The emphasis in our scan protocol was on views that are easily achievable and abnormalities that are easily recognisable for operators with no, or very little, previous ultrasound experience; for example, bladder wall thickening and renal pelvis dilation. More sophisticated procedures such as measurement of bladder volume were omitted. More sophisticated procedures such as measurement of bladder volume were omitted, as well as complex severity grading scores. The initial protocol comprised a transverse scan of the bladder and an oblique and transverse scan of both kidneys. During the course of the study a longitudinal bladder scan was added, given the potential added value in the detection of bladder dome pathology. Bladder abnormalities compatible with UGS were defined as any bladder wall thickening $\geq 5 \mathrm{~mm}$, as in the Niamey criteria, or bladder wall calcification, regardless of kidney and ureter findings. Abnormalities of the kidneys and ureters without bladder pathology were not considered compatible with UGS, as this is a highly uncommon finding and etiologies other than UGS are more likely. A description of the FASUS probe positions is presented in Figure 1. The scan planes and documented variables are presented in Table 1. Examples of target pathologies are presented in Figure 2.

\section{Training}

Two operators, a clinician (JR) and a medical student (AV), with little and no previous ultrasound experience, respectively, were trained on-site. POCUS training took $20 \mathrm{~h}$ overall. A theoretical part included lectures on relevant literature ${ }^{20}$ and demonstration of the FASUS protocol. The practical part consisted of five test scans by each operator in healthy volunteers. Still images and video clips recorded during the five test scans were independently and remotely reviewed by two experts, a pediatric infectious diseases clinician with 4 y (SB) and a clinical radiologist with over $20 \mathrm{y}$ of ultrasound experience (EJ). Detailed feedback on image 


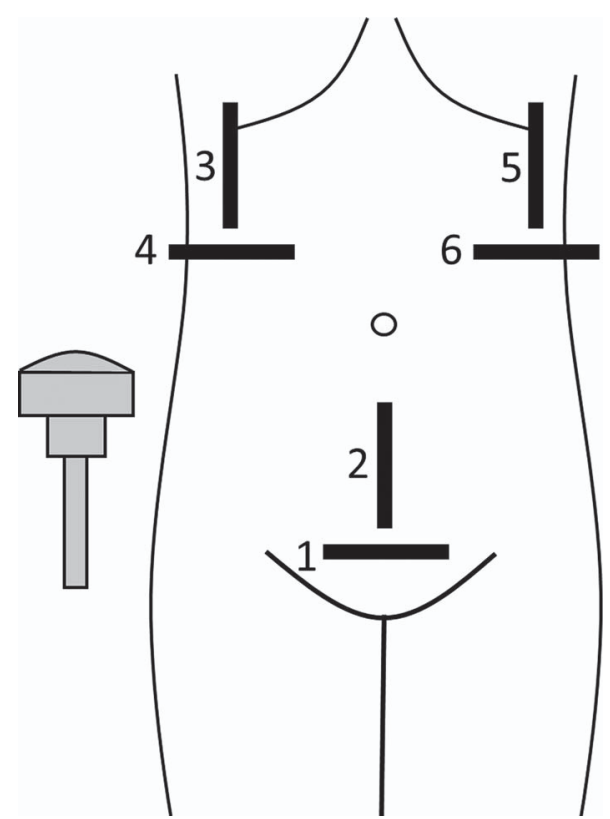

Figure 1. Focused Assessment with Sonography for Urinary Schistosomiasis (FASUS) probe positions: 1 and $2=$ transverse and longitudinal suprapubic pelvis scan; 3 and $4=$ oblique and transverse right kidney scan; 5 and $6=$ oblique and transverse left kidney scan. quality and visualization of target structures was provided, along with practical advice for improvement of technique.

\section{Prospective evaluation of the protocol}

Participants of all ages were recruited by convenience sampling in areas of Lambaréné that were identified as hotspots for UGS in previous studies. ${ }^{16,17}$ Inclusion criteria were previous or ongoing macro-hematuria; exclusion criterion were known diseases of the urinary tract related to causes other than UGS. Convenience sampling was considered appropriate, as this was not intended as a prevalence survey, but as a cohort of patients with clinical symptoms. After an initial clinical assessment, FASUS and urine collection for parasitology were performed. Following praziquantel treatment, active follow-up visits were attempted after 1 and $3 \mathrm{mo}$.

\section{Clinical and laboratory procedures}

Clinical assessment consisted of a questionnaire on present or previous symptoms of UGS, known bladder or kidney disease, previous praziquantel treatment, the duration of fresh water contact and pregnancy. Three urine samples, provided between 10:00 h and 14:00 $\mathrm{h}$ on three consecutive days, were requested. Semiquantitative urine dipstick testing as well as urine filtration $(10 \mathrm{ml}$

Table 1. Focused Assessment with Sonography for Urinary Schistosomiasis (FASUS) protocol

\begin{tabular}{|c|c|c|}
\hline Scan planes & Assessed anatomy & Pathology variables $^{a}$ \\
\hline \multicolumn{3}{|l|}{ Suprapubic pelvis } \\
\hline 1) Transverse & $\begin{array}{l}\text { Bladder wall } \\
\text { Distal ureters }\end{array}$ & $\begin{array}{l}\text { a) Bladder wall irregularities }<5 \mathrm{~mm} \\
\text { thickness } \\
\text { b) Wall thickening } 5-10 \mathrm{~mm} \\
\text { c) Maximum thickening, masses or } \\
\text { pseudopolyps } \geq 10 \mathrm{~mm}\end{array}$ \\
\hline 2) Longitudinal ${ }^{b}$ & $\begin{array}{l}\text { Bladder wall } \\
\text { Distal ureters }\end{array}$ & $\begin{array}{l}\text { d) Bladder wall calcification } \\
\text { e) Distal ureter dilation or thickening } \\
\text { f) Findings not related to urogenital } \\
\text { schistosomiasis }\end{array}$ \\
\hline
\end{tabular}

Kidneys (right and left)

3) and 5) Oblique

4) and 6) Transverse
Proximal ureter and collecting system

Renal pelvis g) Proximal ureter dilation

h) Findings not related to urogenital schistosomiasis

i) Renal pelvis dilation ( $>1 \mathrm{~cm}$ )

- If abnormal, confirmed after bladder voiding

j) Findings not related to urogenital schistosomiasis

a any of b), c) or d) was defined as a pathology compatible with urogenital schistosomiasis.

badded to the protocol after recruitment of 45 cases because a higher sensitivity in the detection of bladder wall thickening was assumed. 


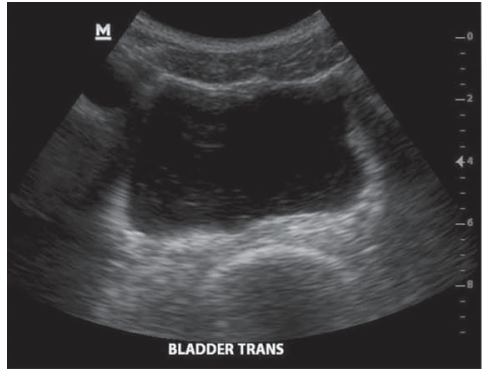

a) Normal bladder

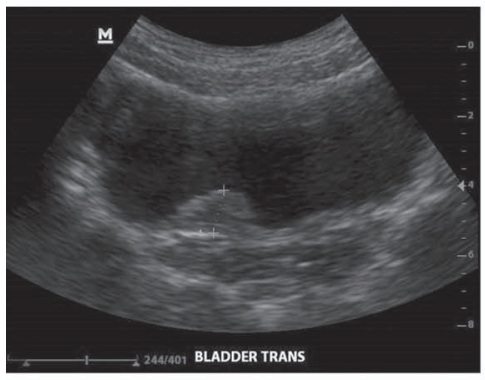

d) Bladder wall thickening $>10 \mathrm{~mm}$

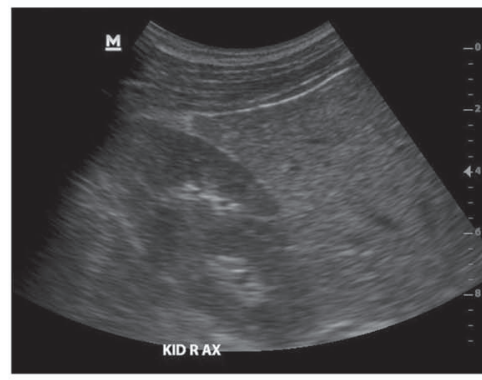

g) Normal kidney

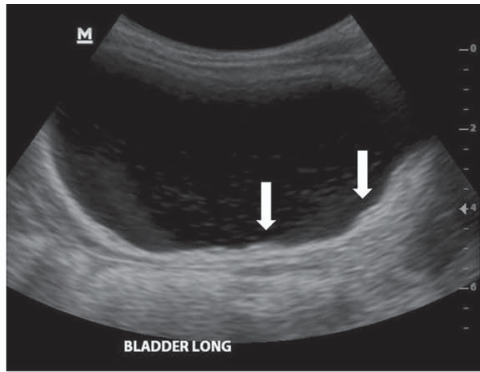

b) Bladder wall irregularities

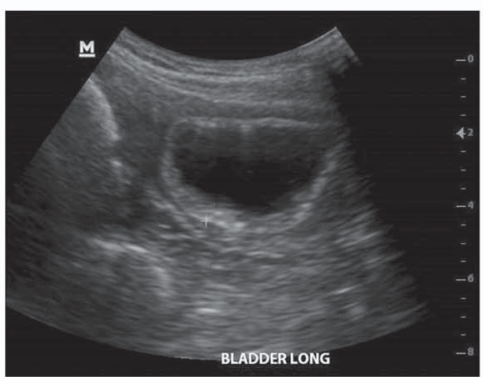

e) Bladder wall calcification

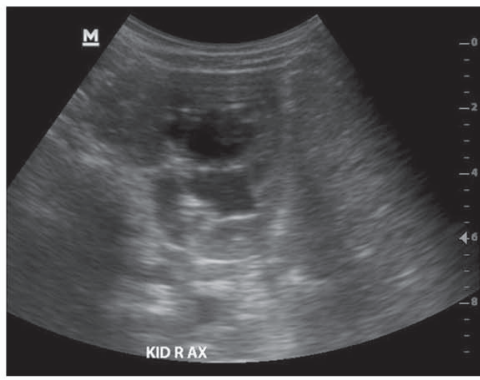

h) Kidney pelvis dilatation

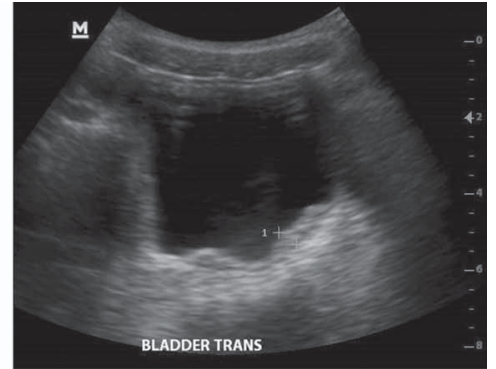

c) Bladder wall thickening $5-10 \mathrm{~mm}$

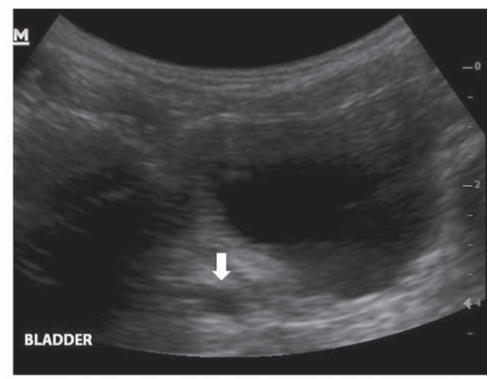

f) Distal ureter dilatation

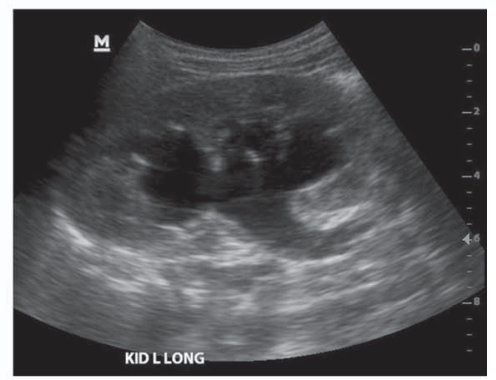

i) Proximal ureter dilatation

Figure 2. Examples of target pathologies detected by Focused Assessment with Sonography for Urinary Schistosomiasis (FASUS).

urine) for microscopy for the presence of Schistosoma haematobium were performed (Dipstick: Combur 10 Test, Roche Diagnostics Ltd, Risch, Switzerland; Millipore filter: Nuclepore TrackEtch Membrane Filtration Products, Whatman, Maidstone, UK; microscope: Eclipse E200MV R, Nikon, Tokyo, Japan). A positive result was defined as any detection of eggs in the filtered urine. Heavy intensity infection was defined as $\geq 50$ eggs $/ 10 \mathrm{ml}$ urine. $^{21}$ In addition, $10 \mathrm{ml}$ of urine was centrifuged for $5 \mathrm{~min}$ at $710 \times \mathrm{g}$ and, after discarding the supernatant, $500 \mu \mathrm{l}$ of urine was stored at $-20^{\circ} \mathrm{C}$ for Schistosoma genus PCR. In the case of negative microscopy, urine real-time PCR for Schistosoma-specific DNA was performed at CERMEL, as published previously. ${ }^{22}$

\section{Treatment and follow-up}

Patients with confirmed UGS by at least one urine sample or by FASUS expert interpretation were treated with a single dose of praziquantel $(40 \mathrm{mg} / \mathrm{kg})$. If non-UGS-related pathology was found in the FASUS examination, the participant was referred for further management.

Follow-up visits took place 1 (M1) and 3 (M3) mo after treatment. Procedures performed during follow-up comprised a clinical investigation, urine analysis by dipstick and microscopy. Patients with FASUS findings suggestive of UGS at enrolment underwent a follow-up scan, which was reviewed and documented similar to enrolment, but using a slightly shorter report form. In cases of persistent signs of urinary tract pathology at $M 3$, retreatment with praziquantel was provided. If advanced UGS-related pathology was not responsive to treatment, the participant was referred to urologic care Gabon's capital Libreville for further management.

\section{FASUS}

FASUS scans were performed and evaluated by one of the two operators (Table 1). FASUS videoclips and images were stored and remotely reviewed and independently interpreted by two ultrasound experts (SB and EJ). This reference test was chosen because cystoscopic evaluation of the bladder as a gold standard would not have been medically or ethically justified, and a locally performed expert reference scan was not feasible in the absence of experts.

A portable low-cost ultrasound device (MINDRAY Digital Ultrasonic Diagnostic Imaging System model DP-10, Mindray Medical 
Table 2. Ultrasound image quality sufficient for expert interpretation

\begin{tabular}{|c|c|c|c|c|c|c|c|}
\hline \multirow[b]{2}{*}{ Ultrasound operator ${ }^{\mathrm{b}}$} & \multirow[b]{2}{*}{ Total scans } & \multicolumn{2}{|c|}{ Bladder scan ${ }^{a}$} & \multicolumn{2}{|c|}{ Right kidney scan } & \multicolumn{2}{|c|}{ Left kidney scan } \\
\hline & & Bladder wall & $\begin{array}{l}\text { Distal } \\
\text { ureters }\end{array}$ & Renal pelvis & $\begin{array}{l}\text { Proximal } \\
\text { ureter }\end{array}$ & Renal pelvis & $\begin{array}{l}\text { Proximal } \\
\text { ureter }\end{array}$ \\
\hline Operator one, n (\%) & $118^{c}$ & $\begin{array}{l}107 \\
(91)\end{array}$ & $\begin{array}{l}100 \\
(88)\end{array}$ & $\begin{array}{l}117 \\
(99)\end{array}$ & $\begin{array}{l}115 \\
(97)\end{array}$ & $\begin{array}{l}115 \\
(97)\end{array}$ & $\begin{array}{l}114 \\
(97)\end{array}$ \\
\hline Operator two, n (\%) & 106 & $\begin{array}{l}95 \\
(90)\end{array}$ & $\begin{array}{l}93 \\
(88)\end{array}$ & $\begin{array}{l}106 \\
(100)\end{array}$ & $\begin{array}{l}103 \\
(97)\end{array}$ & $\begin{array}{l}104 \\
(98)\end{array}$ & $\begin{array}{l}104 \\
(98)\end{array}$ \\
\hline
\end{tabular}

International Limited, Shenzhen, China) with a curved array transducer (MINDRAY model 35C50EB) was used for training as well as for study procedures. Participants underwent FASUS examination either in their homes or at CERMEL following standardized scanning conditions (the same ultrasound equipment, patients in a supine position, a darkened room, the operator seated on the patient's right side). FASUS at enrolment was performed by operator one (JR) and all follow-up scans by operator two (AV). Sufficient bladder filling, defined as complete distention of the bladder, was attempted as a precondition to the examination. Anonymized patient data and ultrasound findings were documented on a clinical record form. Images were stored as digital video clips (Audio Video Interleave (AVI) format) on the ultrasound device; if pathology was suspected, additional still images (JPEG format) were stored. Anonymized data were transferred to an external hard drive, compressed using the open source video transcoder HandBrake (https://ha ndbrake.fr) and bundled into zip files using the program 7zip (http://www.7-zip.org) in order to reduce the file size for upload. Zip files were uploaded to the secure teleradiology platform Collegium Telemedicus (https://collegiumtelemedicus.org) together with basic clinical data (age, gender, current complaints and history).

All images were independently reviewed by the two experts, who were blinded to the operators' findings and laboratory data. Review criteria included the diagnostic quality of the scan (overall image quality, depth, gain, focus, anatomical orientation and sufficient bladder filling) as well as the presence of pathology, as presented in Table 1. The experts documented their findings in an anonymized digital Portable Document Format (PDF) report form. Any discrepant readings between the reviewers were resolved by consensus. For pragmatic purposes, discrepancies in the severity grading of bladder wall pathology were not resolved and only the expert findings of the radiologist EJ are presented here.

\section{Analysis}

Data were entered into OpenClinica version 3.0.4 (OpenClinica, Boston, MA, USA). Data analysis was performed using Microsoft Excel (Microsoft, Redmond, WA, USA).

\section{Results}

\section{Participants}

Between December 2015 and June 2016, 119 participants were enrolled. One patient withdrew consent after enrolment. The cohort comprised 27 (23\%) preschool-age children (PSAC, aged $<6 \mathrm{y}), 56(47 \%)$ school-age children (SAC, aged 6-15 y) and 35 (30\%) adults (aged >15 y); 56/118 (47\%) were females. Microscopic hematuria on dipstick was present in 103/118 (87\%) patients. Urine microscopy was positive for S. haematobium in 105/118 (89\%) patients; 69/118 (58\%) patients were heavily infected. S. haematobium PCR was negative in all 13 patients with negative microscopy. M1 follow-up was performed for a total of 70/118 (59\%) patients, M3 follow-up was performed for a total of $82 / 118$ (69\%) patients. Age and gender distribution of the followup cohort was comparable with the entire study population.

\section{Image quality}

Of a total of 224 FASUS scans, 178 (79\%) were performed at the patients' residences and 46 (21\%) at CERMEL. The proportion of views considered of sufficient quality for diagnostic interpretation is presented in Table 2 . The quality of bladder views was sufficient in $22 / 27$ (81\%) PSAC, 54/56 (96\%) SAC and 31/35 (89\%) adults. Image depth, gain and focus were rated as good in $99 \%, 98 \%$ and $98 \%$ of all scans, respectively. The bladder was entirely displayed in $95 \%$ of the recorded sweeps. Bladder filling was judged sufficient in $86 \%$; in 7 out of 11 bladder scans with insufficient quality, the bladder was underfilled. Anatomical orientation of the kidney scans was good in $92 \%$ with the recorded sweep displaying the renal pelvis in $99 \%$.

\section{Ultrasound findings}

Pathological features compatible with UGS among baseline scans of sufficient quality were documented in 51/118 (43\%) of cases by the operator and in 46/107 (43\%) by the experts, respectively. Frequencies of UGS-related pathologies at baseline by operator and experts are presented in Figure 3. Detection rates of bladder wall pathology, kidney pelvis and proximal ureter dilation were similar between operator and experts. Distal ureter dilation or 


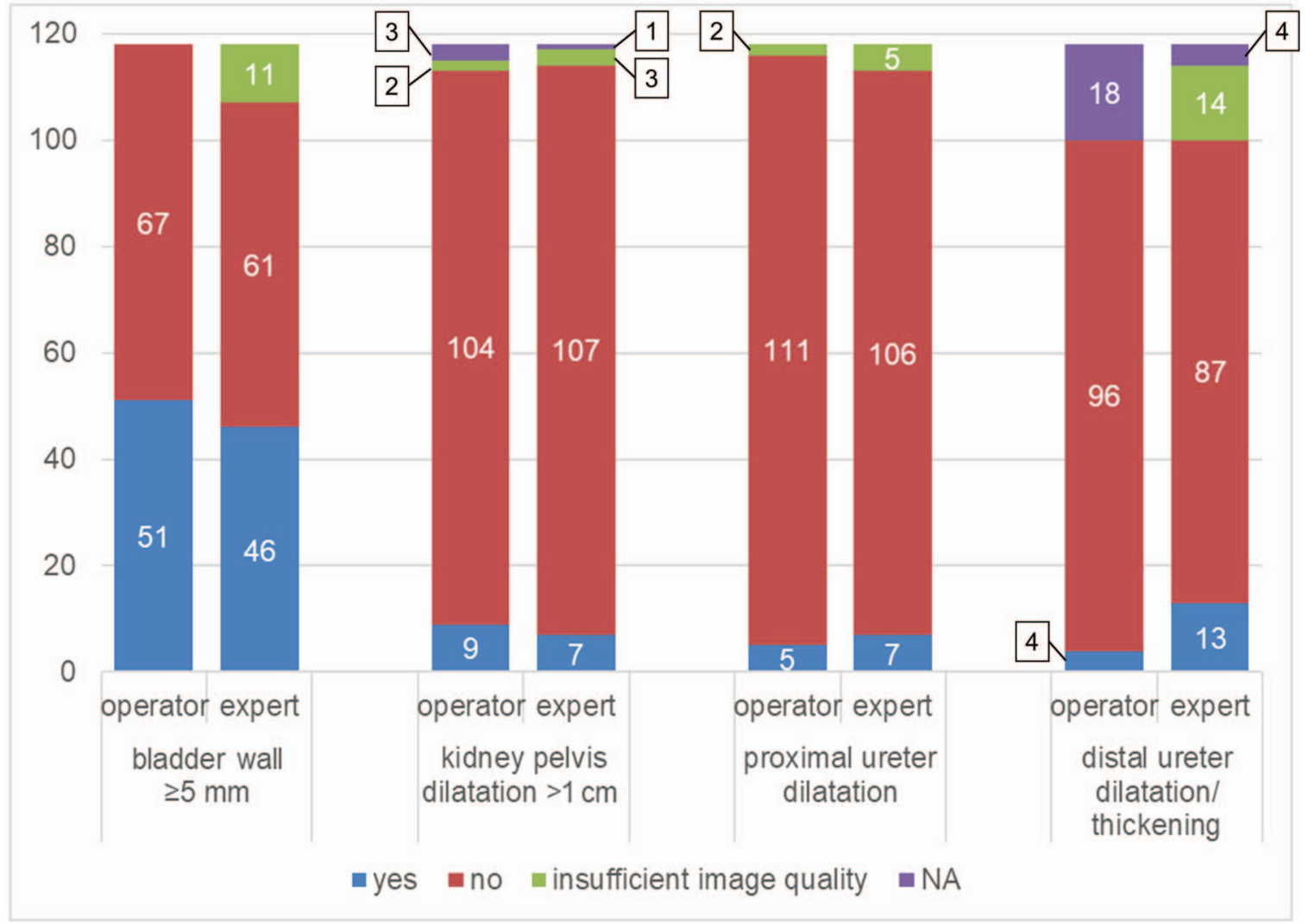

Figure 3. Pathology detected by Focused Assessment with Sonography for Urinary Schistosomiasis (FASUS) at baseline by operator and expert; NA, not available.

thickening was detected three times as often by the experts than by the operator. Frequencies of UGS-related pathologies at baseline as assessed by the experts in relation to parasitology results are presented in Figure 4. With increasing intensity of infection, bladder and upper urinary tract pathology was more frequently detected.

Taking expert findings as the gold standard, the operators missed features compatible with UGS in 21/202 (10\%) cases and overdiagnosed features compatible with UGS in 9/202 (4\%) cases when adding baseline and follow up scans. Sensitivities and specificities of the FASUS operator against the reference standard of a remote expert review are presented in Table 3.

The highest agreement between operators and experts was observed in the detection of renal pelvis dilation, followed by bladder wall thickening of $\geq 5 \mathrm{~mm}$ and proximal ureter dilation. For distal ureter pathology, agreement was lowest for both operators; inter-rater agreement expressed as Cohen's Kappa is presented in Table 3.

The development of image quality, diagnostic accuracy and inter-rater agreement with increasing number of scans is presented in Figure 5 for each operator.

Among 85 cases assessed by both transverse and longitudinal bladder scan, pathology compatible with UGS was detected in 35 (41\%) cases by transverse scan alone compared with 38 (45\%) by a combination of both scans.

In five cases, renal pelvis and/or ureter dilation was present without bladder wall thickening of $\geq 5 \mathrm{~mm}$. One of these patients was pregnant, and in three others bladder wall irregularities were present. All five were positive for S. haematobium in urine microscopy.

Pathology not related to UGS was detected in $1 / 118$ (1\%) patients by the operators and in $9 / 118$ (8\%) patients by the experts and comprised free fluid in the pelvis $(n=3)$, nonspecific kidney lesions $(n=2)$, pregnancy-related hydronephrosis, a urachal remnant, non-specific uterine lesions and non-specific strands in the bladder.

During follow-up, pathology compatible with UGS was present in $15 / 46(33 \%)$ cases at $M 1$ and in $16 / 49(33 \%)$ cases at M3 among scans of sufficient quality. Hydronephrosis was present in $3 / 51(6 \%)$ and $3 / 54(6 \%)$ cases at M1 and M3, respectively. As criteria for follow-up were not consistent, these rates cannot be used to measure treatment success.

\section{Discussion}

This pilot study was the first to evaluate a POCUS protocol for the detection of UGS-related pathology of the urinary tract in symptomatic patients living in a highly endemic area. It showed that POCUS operators with limited training can detect such pathology with high accuracy, compared with expert ultrasound operators. Inter-rater agreement between operator and expert was good for the detection of bladder wall pathology and very good for hydronephrosis. Image quality was sufficient in more than $88 \%$ of all scans. 


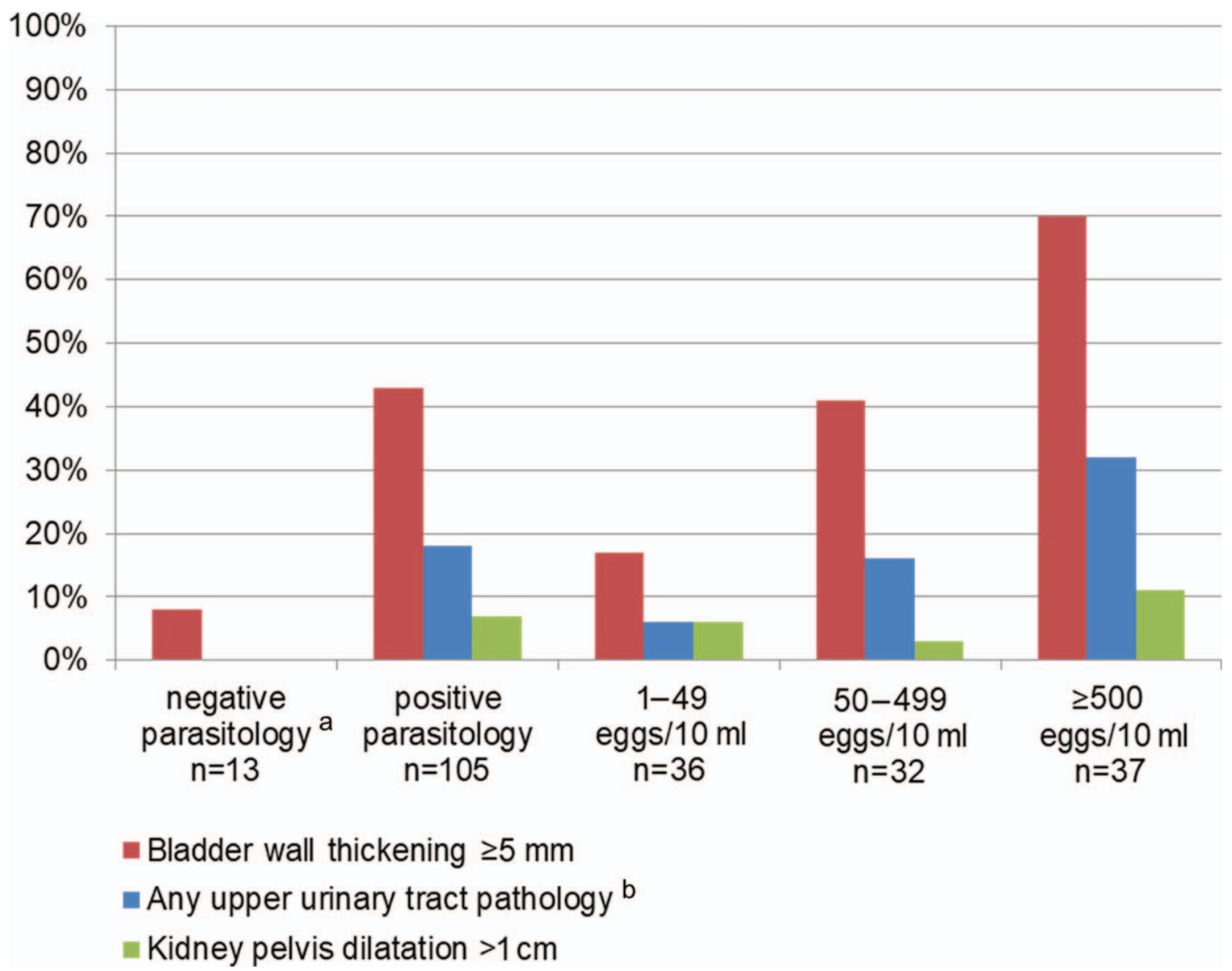

Figure 4. Pathology detected by Focused Assessment with Sonography for Urinary Schistosomiasis (FASUS) at baseline (expert review) in relation to parasitology; ${ }^{a}$ urine microscopy and PCR; ${ }^{b}$ renal pelvis dilation $>1 \mathrm{~cm}$, proximal ureter dilation or distal ureter dilation/thickening.

Table 3. Accuracy of newly trained operators, compared to expert ultrasound opinion

\begin{tabular}{|c|c|c|c|c|c|c|c|c|}
\hline \multirow{2}{*}{$\begin{array}{l}\text { Accuracy }^{a} \\
\text { Operator }^{c}\end{array}$} & \multicolumn{2}{|c|}{$\begin{array}{l}\text { Bladder wall thickening } \\
\qquad \geq 5 \mathrm{~mm}\end{array}$} & \multicolumn{2}{|c|}{$\begin{array}{l}\text { Distal ureter dilation/ } \\
\text { thickening }\end{array}$} & \multicolumn{2}{|c|}{$\begin{array}{l}\text { Proximal ureter } \\
\text { dilation }\end{array}$} & \multicolumn{2}{|c|}{$\begin{array}{l}\text { Renal pelvis } \\
\text { dilation }>1 \mathrm{~cm}^{\mathrm{b}}\end{array}$} \\
\hline & 1 & 2 & 1 & 2 & 1 & 2 & 1 & 2 \\
\hline Total scans ${ }^{d}$ & 107 & 95 & 87 & 93 & 111 & 102 & 110 & 104 \\
\hline Sensitivity (\%) & 83 & 68 & 17 & 23 & 57 & 50 & 100 & 83 \\
\hline Specificity (\%) & 90 & 95 & 97 & 99 & 100 & 95 & 98 & 100 \\
\hline $\begin{array}{l}\text { Observed inter- } \\
\text { rater agreement }\end{array}$ & 0.87 & 0.83 & 0.86 & 0.88 & 0.97 & 0.94 & 0.98 & 0.99 \\
\hline Cohen's Kappa & $\begin{array}{l}0.73 \\
\text { "good" }\end{array}$ & $\begin{array}{l}0.64 \\
\text { "good" }\end{array}$ & $\begin{array}{l}0.19 \\
\text { "poor" }\end{array}$ & $\begin{array}{l}0.31 \\
\text { "fair" }\end{array}$ & $\begin{array}{c}0.71 \\
\text { "good" }\end{array}$ & $\begin{array}{l}0.23 \\
\text { "fair" }\end{array}$ & $\begin{array}{c}0.85 \\
\text { "very good" }\end{array}$ & $\begin{array}{c}0.90 \\
\text { "very good" }\end{array}$ \\
\hline
\end{tabular}

a Ultrasound operator findings compared to expert consensus after remote review of recorded clips.

${ }^{b}$ If confirmed after bladder voiding, or if severe.

'Baseline scans performed by operator one, follow up scans performed by operator two.

${ }^{\mathrm{d}}$ Number of scans of sufficient image quality for interpretation by operator and expert consensus. 


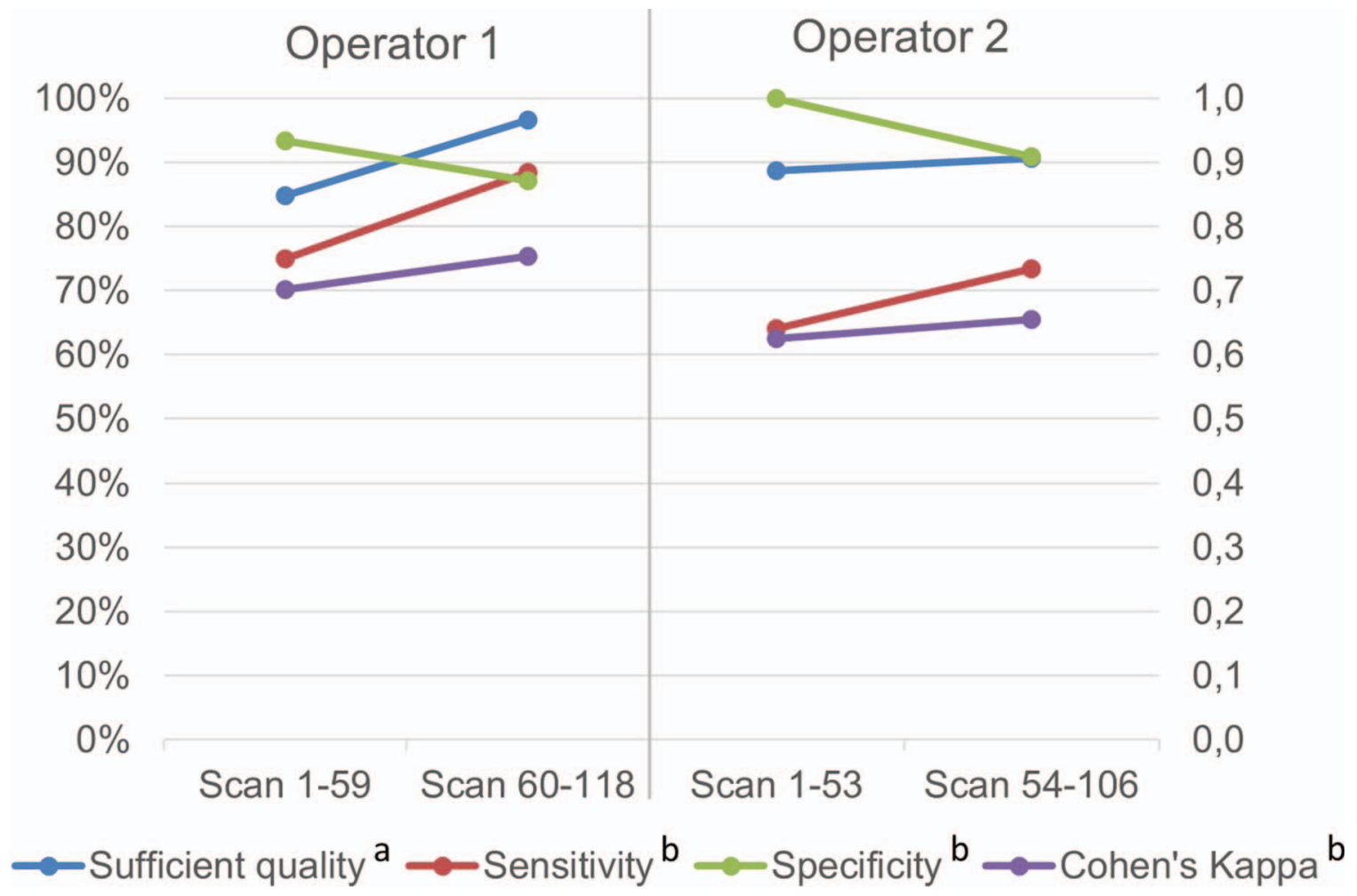

Figure 5. Operator improvement in bladder wall assessment; ${ }^{a}$ image quality of bladder scans sufficient for expert interpretation; ${ }^{b}$ ultrasound operator findings compared with expert consensus after remote review of recorded clips.

\section{Protocol design}

In our protocol, pathology compatible with UGS was defined as bladder wall thickening $\geq 5 \mathrm{~mm}$. This cut-off was chosen based on existing Niamey criteria. However, in many cases bladder wall irregularities of $<5 \mathrm{~mm}$ were detected, indicating that less advanced pathology, compatible with UGS, can also be detected with current equipment and short, focused training. This finding may be of value, as an earlier detection of morbidity may influence management decisions. Differentiation of bladder wall irregularities from a normal bladder wall remains challenging, as illustrated by Figure 2 and reported in other studies. ${ }^{10}$ Therefore, a threshold of $5 \mathrm{~mm}$ appears sensible to limit false positive cases. To keep the protocol as pragmatic as possible for use in the clinical setting, a classification of pathology severity, combining bladder and upper urinary tract pathology, as applied in other studies, ${ }^{5,23}$ was not used. However, as upper urinary tract pathology with concurrent bladder wall irregularities of less than $5 \mathrm{~mm}$ is also likely to be due to UGS (although classified as 'not compatible with UGS' in our study), this approach needs to be reconsidered in larger validation studies.

As expected, the operators detected fewer abnormalities not related to UGS. The aim of POCUS is to answer a very specific clinical question, in this case: 'Are there findings compatible with UGS?' and not to provide a comprehensive ultrasound examination or to exclude the presence of other pathology.

While the Niamey protocol ${ }^{12}$ comprises only a transverse view of the bladder, we added a longitudinal bladder view assuming a higher sensitivity for the detection of pathology, especially at the bladder dome. With three additional cases of bladder pathology detected by the additional longitudinal view, we recommend including this view in further evaluations of this protocol.

\section{Training}

During training and data collection, the team was repeatedly approached by local healthcare staff willing to learn the method, demonstrating the general interest in the achievement of ultrasound skills among local clinicians.

Remote training support and image assessment proved effective in attaining good image quality, but as it comprised only scans in healthy volunteers, identification of pathology could be learnt in theory only. An electronic learning module with quiz-like elements could resolve this deficit. Although remote feedback was given for study patient scans as well, it was staggered and therefore time-delayed. The optimal number of training scans for FASUS needs to be determined. A broad spectrum of training requirements for point-of-care ultrasound protocols, ranging from a few hours to several days, has been proposed depending on sonographic complexity and previous ultrasound knowledge..$^{24-26}$

\section{Image quality}

Overall image quality was very good with more than $90 \%$ of bladder walls and more than $97 \%$ of kidneys and proximal ureters 
being assessable by the experts. The display of distal ureters was slightly more challenging. The high expert ratings on gain, depth and focus settings show the effectiveness of the chosen remote training model.

Image quality was best among SAC due to excellent displays of deep anatomical structures related to smaller size. Inferior image quality among PSAC and some adults can be explained by limited compliance and additional body fat, respectively.

Interestingly, only two kidney scans and no bladder scans were rated as 'unable to assess' by operator one, and only two bladders and one kidney scan by operator two, showing that the operators attempted to make assessments, despite limitations in image quality. This is a potential source of error and future training should emphasize this risk.

As reported in other studies, ${ }^{10}$ sufficient bladder filling is crucial for the quality of bladder scans. In the absence of a definition or measurement for sufficient bladder filling, in our study insufficient bladder filling was judged by eyeballing bladder content and wall. For future application of FASUS, a clear definition of sufficient or insufficient bladder filling would be valuable; however, including measurements of bladder volumes for age-related normal ranges would considerably increase complexity. Learning the critical point of insufficient bladder filling needs to be part of FASUS training.

\section{Accuracy of pilot operators compared with experts}

Overall, the accuracy of FASUS, performed by our pilot operators, was good for the detection of bladder wall thickening $\geq 5 \mathrm{~mm}$ and very good for the detection of hydronephrosis. Accuracy for the detection of ureter pathology was lower, with $8 / 10$ and 5/6 cases of distal ureter pathology missed by the two operators, respectively. As all cases of distal ureter pathology occurred together with bladder wall pathology, the additional diagnostic value of distal ureter assessment needs to be questioned. Considering its difficulty, it should be omitted from the protocol.

While image quality and sensitivity improved for both operators over the course of the study, there was a slight decline in specificity. Although conclusions need to be treated with caution due to the small sample size, this might point to a shift from underestimation to overestimation of pathology with an increasing number of scans.

In another study on the learning curve of the more complicated Niamey protocol for prevalence surveys by Bonnard et al., sensitivity and specificity of the learner in comparison with the teacher had reached 100\% for both after $5 \mathrm{~d}$ and 91 examinations. ${ }^{27}$ However, the teacher directly supervised the learner, in contrast to the staggered remote feedback over the period of around 3 mo per operator in our study. Furthermore, the authors were unclear whether the learning clinician had previous ultrasound experience. Two other studies on remotely reviewed POCUS in tropical medicine describing the detection of extrapulmonary TB provided a sensitivity and specificity of the operator compared with the expert that was comparable to our study. ${ }^{28,29}$ However, unlike our approach, the examinations were transmitted and assessed remotely in real time and not via saved video clips, thus limiting comparison.
As urinary tract pathology increased with increasing intensity of infection in this as well as in other studies, ${ }^{30}$ an algorithm which combines parasitological with ultrasonographic findings, especially in patients with a heavy intensity of infection, could maximize diagnostic accuracy.

Overall, the learning of this POCUS technique via remote review and feedback appears to be feasible and leads to good accuracy that can be maintained over longer periods.

Of note, as the intention of this study was not to identify infection rates, the reported rate of UGS-related pathology among the study population does not reflect that in the general population in Gabon.

\section{Management of pathological findings}

As sonographic detection of UGS-related pathology has mostly been used in the evaluation of large-scale control programs, no algorithms for individual patient management based on ultrasound are available. FASUS could help in the identification of at-risk patients in need of regular follow-up and retreatment in areas where regular preventive treatment is not indicated or insufficiently implemented. Patients with advanced pathology such as therapy-resistant hydronephrosis or suspicious bladder lesions could be referred for further diagnostics and management, where available.

\section{Limitations}

As a pilot study, the protocol underwent changes during the course of the study, thus parts of the data are incomplete.

Our reference test of a remote expert review is inferior to a locally performed expert reference scan, but was the best and only feasible option in the absence of local expertise. With only quality-reduced clips and still images as a basis for the interpretation, pathology might have been missed by the experts or normal findings and artifacts mistaken for pathology. However, this telemedicine-based training and image review design provides a feasible model for POCUS training in resource-limited settings where no alternative options exist.

\section{Conclusions}

FASUS is a promising bedside diagnostic tool for detection of UGS-related morbidity in symptomatic patients living in highly endemic areas. Simple protocols and short training appear adequate and feasible, despite the common limitations present in a low-resource setting, such as novice clinician trainees, lowbudget ultrasound devices and examinations performed in the field. If further validation studies confirm the utility of FASUS, internationally agreeable standards for training and competency should be set. Based on data from larger cohorts, appropriate diagnostic and therapeutic algorithms for its use in routine patient care should be established.

\section{Authors' contributions}

JR, ALB, TH, EJ and SB conceived the study and JR, ALB, TH, AAA, MPG, MR, EJ and SB designed the study protocol; JR and AV carried out the ultrasonographic assessments; EJ and SB performed the 
digital review of ultrasound images. AV, NGT and GPM carried out the laboratory tests; JR, AV and RO performed the data analysis. JR, ALB, TH, EJ and SB drafted the manuscript; all authors critically revised the manuscript and contributed to and approved the final version.

\section{Funding}

There was no dedicated funding for the study. SB is currently a participant in the BIH-Charite Clinician Scientist Program funded by Charité-Universitätsmedizin Berlin and the Berlin Institute of Health.

\section{Competing interests}

None declared.

\section{Ethical approval}

This study was approved by the scientific review committee and the institutional ethics committee of CERMEL. Written informed consent was obtained from all patients (or legal guardians in the case of children) prior to study enrolment.

\section{Acknowledgements}

We thank Collegium Telemedicus for the provision of their teleradiology platform.

\section{References}

1 WHO. Schistosomiasis - Epidemiology.2019. https://www.who.i nt/schistosomiasis/epidemiology/table/en/ (accessed 12 February 2019).

2 Colley DG, Bustinduy AL, Secor WE et al. Human schistosomiasis. Lancet 2014;383:2253-2264.

3 Barda B, Coulibaly JT, Hatz C et al. Ultrasonographic evaluation of urinary tract morbidity in school-aged and preschool-aged children infected with Schistosoma haematobium and its evolution after praziquantel treatment: a randomized controlled trial. PLoS Negl Trop Dis 2017;11:e0005400.

4 Murray CJ, Barber RM, Foreman KJ, et al. Global, regional, and national disability-adjusted life years (DALYs) for 306 diseases and injuries and healthy life expectancy (HALE) for 188 countries, 1990-2013, quantifying the epidemiological transition. Lancet 2015;386:2145-91

5 Hatz CF, Vennervald BJ, Nkulila T et al. Evolution of Schistosoma haematobium-related pathology over 24 months after treatment with praziquantel among school children in southeastern Tanzania. Am J Trop Med Hyg 1998;59:775-781.

6 Correia da Costa JM, Vale N, Gouveia MJ et al. Schistosome and liver fluke derived catechol-estrogens and helminth associated cancers. Front Genet 2014;5:444.

7 Koukounari A, Webster JP, Donnelly CA et al. Sensitivities and specificities of diagnostic tests and infection prevalence of Schistosoma haematobium estimated from data on adults in villages northwest of Accra, Ghana. Am J Trop Med Hyg 2009;80:435-441.

8 Weerakoon KG, Gobert GN, Cai P et al. Advances in the diagnosis of human Schistosomiasis. Clin Microbiol Rev 2015;28:939-967.

9 Chernet A, Kling K, Sydow V et al. Accuracy of diagnostic tests for Schistosoma mansoni infection in asymptomatic Eritrean refugees: serology and point-of-care circulating Cathodic antigen against stool microscopy. Clin Infect Dis 2017;65:568-574.

10 Akpata R, Neumayr A, Holtfreter MC et al. The WHO ultrasonography protocol for assessing morbidity due to Schistosoma haematobium. Acceptance and evolution over 14 years. Systematic review. Parasitol Res 2015;114:1279-1289.

11 Santos J, Chaves J, Araujo $\mathrm{H}$ et al. Comparison of findings using ultrasonography and cystoscopy in urogenital schistosomiasis in a public health centre in rural Angola. S Afr Med J 2015;105: 312-315.

12 WHO, ULTRASOUND IN SCHISTOSOMIASIS. A Practical Guide to the Standardized Use of Ultrasonography for the Assessment of Schistosomiasis-related Morbidity. In: Richter $J$ et al., editors. TDR/STR/SCH/00.1, 1996, WHO Press, World Health Organization, Geneva, Switzerland.

13 Belard S, Tamarozzi F, Bustinduy AL et al. Point-of-care ultrasound assessment of tropical infectious diseases-a review of applications and perspectives. Am J Trop Med Hyg 2016;94:8-21.

14 WHO. Gabon Country Profile. 2010. www.who.int/neglected_disease s/preventive_chemotherapy/.../CP_Gabon.pdf (accessed 12 February 2019).

15 Adegnika AA, Ramharter M, Agnandji ST et al. Epidemiology of parasitic co-infections during pregnancy in Lambarene, Gabon. Trop Med Int Health 2010;15:1204-1209.

16 Ateba Ngoa U, Zinsou JF, Kassa RF et al. Assessment of the effect of Schistosoma haematobium co infection on malaria parasites and immune responses in rural populations in Gabon: study protocol. Springerplus 2014;3:388.

17 Dejon-Agobe JC, Zinsou JF, Honkpehedji YJ et al. Schistosoma haematobium effects on plasmodium falciparum infection modified by soiltransmitted helminths in school-age children living in rural areas of Gabon. PLoS Negl Trop Dis 2018;12:e0006663.

18 Mombo-Ngoma G, Honkpehedji J, Basra A et al. Urogenital schistosomiasis during pregnancy is associated with low birth weight delivery: analysis of a prospective cohort of pregnant women and their offspring in Gabon. Int J Parasitol 2017;47:69-74.

19 Basra A, Mombo-Ngoma G, Melser MC et al. Efficacy of mefloquine intermittent preventive treatment in pregnancy against Schistosoma haematobium infection in Gabon: a nested randomized controlled assessor-blinded clinical trial. Clin Infect Dis 2013;56:e68-e75.

20 WHO. Manual of Diagnostic Ultrasound. Vol. 1, 2nd edition. WHO Press, World Health Organization, Geneva, Switzerland, 2011.

21 WHO The Control of Schistosomiasis. Report of a WHO Expert Committee. World Health Organization Technical Report Series 1985;728:1-113.

22 Kenguele HM, Adegnika AA, Nkoma AM et al. Impact of short-time urine freezing on the sensitivity of an established Schistosoma realtime PCR assay. Am J Trop Med Hyg 2014;90:1153-1155.

23 Wagatsuma Y, Aryeetey ME, Sack DA et al. Resolution and resurgence of Schistosoma haematobium-induced pathology after communitybased chemotherapy in Ghana, as detected by ultrasound. J Infect Dis 1999;179:1515-1522.

24 Bonnard P, Boutouaba S, Diakhate I et al. Learning curve of vesicourinary ultrasonography in Schistosoma haematobium infection with WHO practical guide: A "simple to learn" examination. Am J Trop Med Hyg 2011;85:1071-1074.

25 Heller T, Wallrauch C, Lessells RJ et al. Short course for focused assessment with sonography for human immunodeficiency virus/tuberculosis: Preliminary results in a rural setting in South Africa with high prevalence of human immunodeficiency virus and tuberculosis. Am J Trop Med Hyg 2010;82:512-515.

26 Shah VP, Tunik MG, Tsung JW. Prospective evaluation of point-ofcare ultrasonography for the diagnosis of pneumonia in children and young adults. JAMA Pediatr 2013;167:119-125. 
27 Bonnard P, Boutouaba S, Diakhate I et al. Learning curve of Vesicourinary ultrasonography in Schistosoma haematobium infection with WHO practical guide: a "simple to learn" examination. Am J Trop Med Hyg 2011;85:1071-1074.

28 Janssen S, Basso F, Giordani MT et al. Sonographic findings in the diagnosis of HIV-associated tuberculosis: Image quality and interobserver agreement in FASH vs. remote-FASH ultrasound. J Telemed Telecare 2013;19:491-493.
29 Janssen S, Grobusch MP, Heller T. 'Remote FASH' telesonography - a novel tool to assist diagnosing HIV-associated extrapulmonary tuberculosis in remote areas. Acta Trop 2013;127: 53-55.

30 Koukounari A, Sacko M, Keita AD et al. Assessment of ultrasound morbidity indicators of schistosomiasis in the context of large-scale programs illustrated with experiences from Malian children. Am J Trop Med Hyg 2006;75:1042-1052. 